\title{
Usefulness of a Long-Straight Sheath for Atrial Lead Insertion at the Right Atrial Septum
}

\author{
Nobuhiro Nishii, MD; Kengo Fukushima Kusano, MD; Kohei Miyaji, MD; \\ Shigeki Hiramatsu, MD; Takeshi Tada, MD; Masato Murakami, MD; \\ Kimikazu Banba, MD; Yoshiaki Sakai, MD; Satoshi Nagase, MD; \\ Kazufumi Nakamura, MD; Satoru Sakuragi, MD; Tohru Ohe, MD
}

\begin{abstract}
Background Pacing at the right atrial septum (RAS) appears to be superior to that performed at the right atrial appendage for preventing paroxysmal atrial fibrillation and for reducing complications. However, atrial lead insertion at the RAS is difficult and time-consuming. Although a few useful devices have been reported, handling of the atrial lead around the RAS is sometimes difficult even with those devices. Therefore, in the present study a long-straight sheath (LSS) was used in an attempt to secure sufficient space for handling the atrial lead around the RAS.

Methods and Results Seventy-six consecutive patients who underwent insertion of an atrial lead at the RAS from a left-sided approach were examined. The non-LSS group comprised 24 patients and the LSS group had 52. The success rate of atrial lead insertion was significantly higher in the LSS group than in the non-LSS group [50/52 $(96.1 \%)$ vs $13 / 24(54.2 \%) ; \mathrm{p}<0.01]$. The operation time was significantly shorter in the LSS group $(13.6 \pm 9.1 \mathrm{~min}$ vs $32.7 \pm 11.1 \mathrm{~min}, \mathrm{p}<0.01)$. P wave duration was significantly shorter during RAS pacing than during normal sinus rhythm.
\end{abstract}

Conclusion The LSS technique is useful for atrial lead insertion at the RAS because it enables sufficient space to be secured for lead handling. (Circ J 2008; 72: 262-267)

Key Words: Atrial fibrillation; Atrial septal pacing; Bachmann's bundle; Long-straight sheath

$\mathbf{N}$ umerous retrospective and prospective studies have shown favorable results for chronic atrial pacing for the prevention of paroxysmal atrial fibrillation (PAF) $)^{1-6}$ It has also been reported that pacing at the right atrial septum (RAS) appears to be superior to that performed at the right atrial appendage (RAA) or free wall for preventing PAF with sinus bradycardia ${ }^{7-12}$ and for reducing complications such as pericarditis, diaphragmatic stimulation, perforation, cardiac tamponade and pneumothorax?,13-21 However, insertion of the lead at the RAS is sometimes technically difficult and time-consuming.

Recently, a few useful devices have been proposed [Locator ${ }^{\mathrm{TM}}$ (St Jude Medical, Sylmar, CA, USA)] and the modified J stylet. De Voogt et al reported good results for the use of a Locator ${ }^{\mathrm{TM}}$, but far field R wave (FFRW) sensing shows only a trend in being higher in the low RAS than in the RAA22 Bailin et al reported a technique of Bachmann's bundle pacing using a modified $\mathrm{J}$ stylet, ${ }^{23}$ targeting Bachmann's bundle, which is in the anterior RAS (near the tricuspid valve), may occasionally result in

(Received August 26, 2007; revised manuscript received October 11, 2007; accepted October 22, 2007)

Department of Cardiovascular Medicine, Okayama University Graduate School of Medicine, Dentistry, and Pharmaceutical Sciences, Okayama, Japan

Mailing address: Nobuhiro Nishii, MD, Department of Cardiovascular Medicine, Okayama University Graduate School of Medicine, Dentistry, and Pharmaceutical Sciences, 2-5-1 Shikata-cho, Okayama 700-8558, Japan. E-mail: gmd14009@cc.okayama-u.ac.jp or nnnnishii2001@ yahoo.co.jp

All rights are reserved to the Japanese Circulation Society. For permissions, please e-mail: cj@j-circ.or.jp serious complications such as cardiac tamponade or aortic injury? ${ }^{24}$ An alternative site for atrial lead insertion is the posterior RAS (posterior Bachmann's bundle), which is near the superior vena cava and distant from the tricuspid valve or the aorta, but handling of the lead in the small space around the posterior RAS is very difficult, even if a Locator $^{\mathrm{TM}}$ or modified $\mathbf{J}$ stylet is used. After several efforts, we have found that an easy approach to handling the atrial leads in the small space around the posterior RAS is to use a long-straight sheath (LSS) with a left-sided approach, with the tip of the LSS near the free wall and distant from the RAS. Accordingly, in the present study we compared the usefulness of the LSS method with that of the standard method for fixing an atrial lead at the posterior RAS.

\section{Methods}

\section{Patients}

The subjects were 76 consecutive patients in whom atrial lead insertion at the posterior RAS was attempted in Okayama University Hospital during the period from November 2004 to November 2006. In the first period (Nov 1, 2004 to Jul 31, 2005), the LSS was not used (nonLSS group: $n=24$ ), but it was in the second period (Aug 1, 2005 to Nov 30, 2006) (LSS group: $\mathrm{n}=52$ ). All procedures were performed from a left-sided approach.

\section{Parameters}

We evaluated the success rate and operation time of atrial lead insertion at the RAS. The latter was defined as the time from the start of atrial lead insertion to measurement of the electrical parameters after screwing in the lead. 


\section{F long-straight sheath $(25 \mathrm{~cm})$}
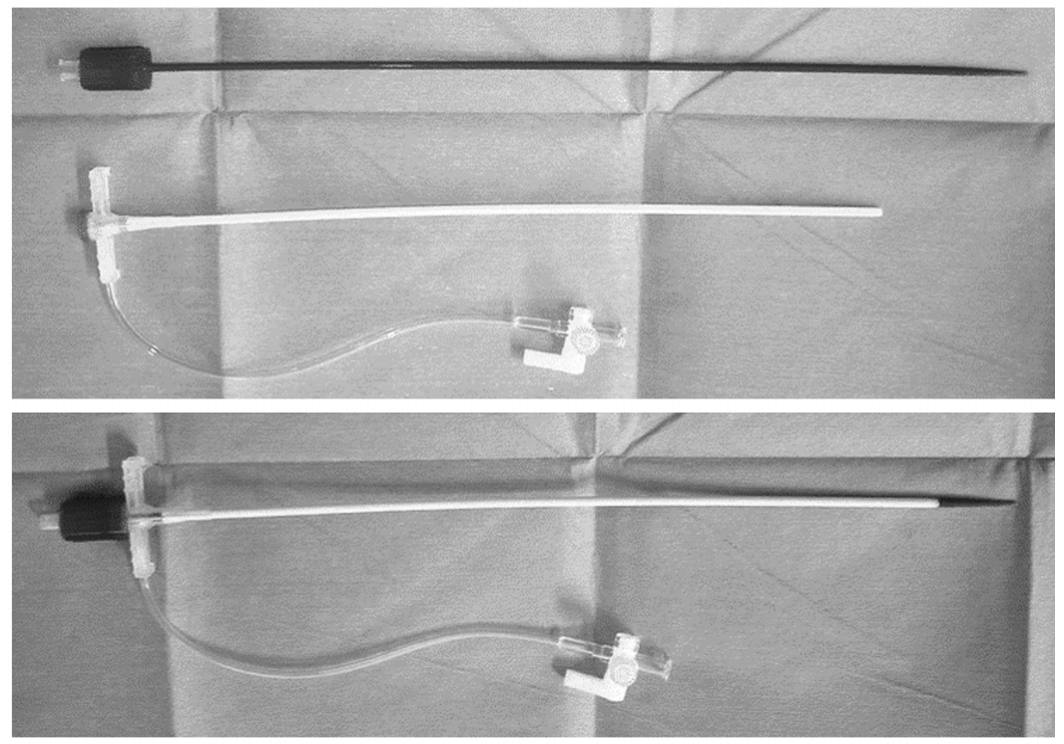

Fig 1. An $8 \mathrm{~F}$ peel-away, long-straight sheath (long-straight sheath: Medikit Peel-off Introducer, $25 \mathrm{~cm}$ and $30 \mathrm{~cm}$, MEDIKIT Co Ltd, Tokyo, Japan), which is soft and flexible, but kink-resistant.
We also evaluated acute $\mathrm{P}$ wave amplitude, pacing threshold, lead impedance and $\mathrm{P}$ wave duration during RAS pacing or normal sinus rhythm (NSR) on 12-lead ECG in the LSS and non-LSS groups.

\section{LSS}

In the LSS group, we used a peel-away 8F LSS (Medikit Peel-off Introducer, 25 or $30 \mathrm{~cm}$, Medikit Co Ltd, Tokyo, Japan) (Fig 1), because it is soft and flexible, but kink-resistant. Therefore, the LSS was able to safely follow the curved junction of the superior vena cava and the left brachiocephalic vein.

\section{Stylet}

We mainly used a manually bent stylet (Fig 2A) with a smaller curve than that of a standard curved stylet for RAA insertion (Fig 2B).

\section{Atrial Lead Insertion Procedure}

First, we slowly inserted the LSS at the mid right atrium (RA) position, taking care to avoid venous or cardiac injury, especially in the curved junction of the superior vena cava and the left brachiocephalic vein, as with lead insertion. The tip of the LSS was then located laterally and posteriorly. In this position, sufficient space between the tip of the LSS and the posterior RAS was obtained (Figs 3A, $\mathrm{C}, \mathrm{D}, \mathrm{F}$ ) and we could easily manipulate the atrial lead in the RA towards the RAS.

In contrast, using the right-sided approach, the tip of the LSS is near the septum and distant from the free wall, and sufficient space for handling of the lead near the RAS cannot be obtained. Therefore, patients in whom this approach was used were excluded from the study.

Next, we introduced an active fixation lead into the RA through the LSS and exchanged the straight stylet for the curved type. We carefully inserted the atrial lead at the posterior RAS (posterior Bachmann's bundle) to avoid Koch's triangle, the free wall and roof of the RA (Fig 4).

First, with the fluoroscope in the 30-degree, right anterior oblique position, the lead was rotated toward the posterior RAS in a counterclockwise direction, taking care to

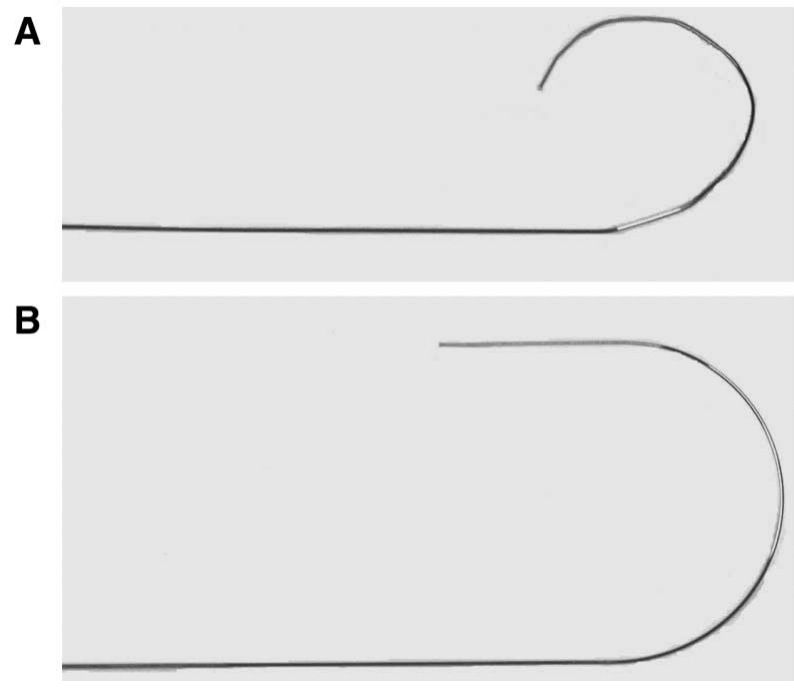

Fig 2. (A) Manually bent stylet with a smaller curve than that of the standard stylet for right atrial appendage (RAA) insertion. (B) Standard stylet for RAA insertion.

avoid the area near the tricuspid valve and the anterior roof (Figs 3B, 4). Next, we confirmed that the lead was directed toward the RAS on a 40- or 50-degree left anterior oblique view (Fig 3E). After the lead was fixed, acute lead sensing and pacing threshold tests were performed. Fig 3 is representative mages of the atrial lead inserted at the posterior RAS (Figs 3B, E).

When we could not fix the lead at the posterior RAS or could not obtain a P-wave amplitude of more than $1.5 \mathrm{mV}$ and a pacing threshold of less than $1.5 \mathrm{~V}$ at $0.5 \mathrm{~ms}$, we inserted the atrial lead at the RAA instead of the RAS.

\section{Atrial Leads}

All the atrial leads that we attempted to insert at the RAS were active fixation leads (1488T, 1688T, St Jude Medical; 5068, 5076, 6940, Medtronic Inc, Minneapolis, MN, USA; 4470, 4471, Guidant Corp, St Paul, MN, USA). 
RAO $30^{\circ}$

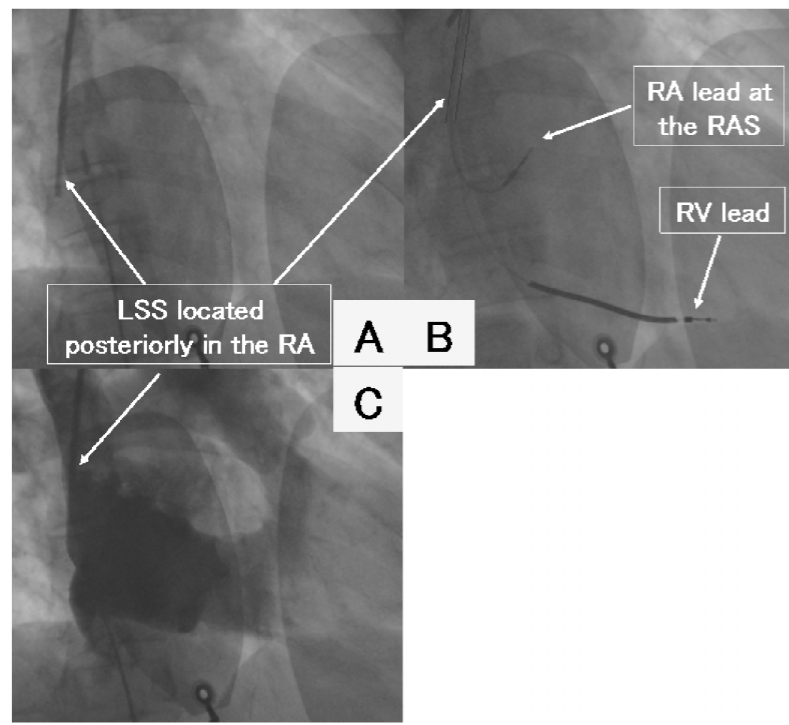

\section{LAO $50^{\circ}$}

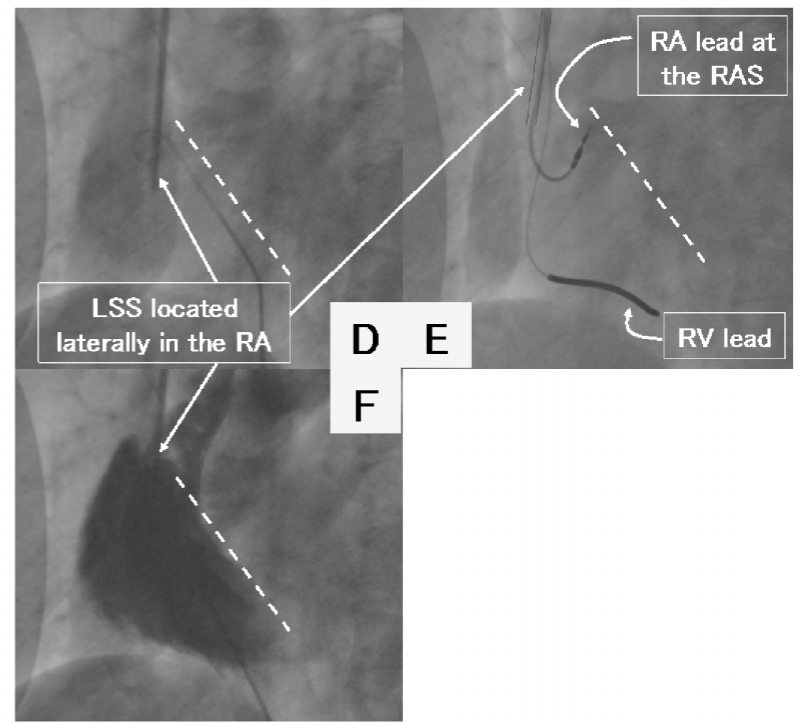

Fig 3. The atrial lead is inserted at the posterior right atrial septum (RAS) (posterior Bachmann's bundle). (A-C) Right anterior oblique (RAO) views, (D-F) left anterior oblique (LAO) views. (C, F) Right atriography. The tip of the longstraight sheath (LSS) is located laterally and posteriorly in the right atrium (RA) (A, C, D, F). The atrial lead is screwed at the posterior RAS (posterior Bachmann's bundle) perpendicularly (B, E). The dashed line (D, E, F) shows that the RAS is sloped.

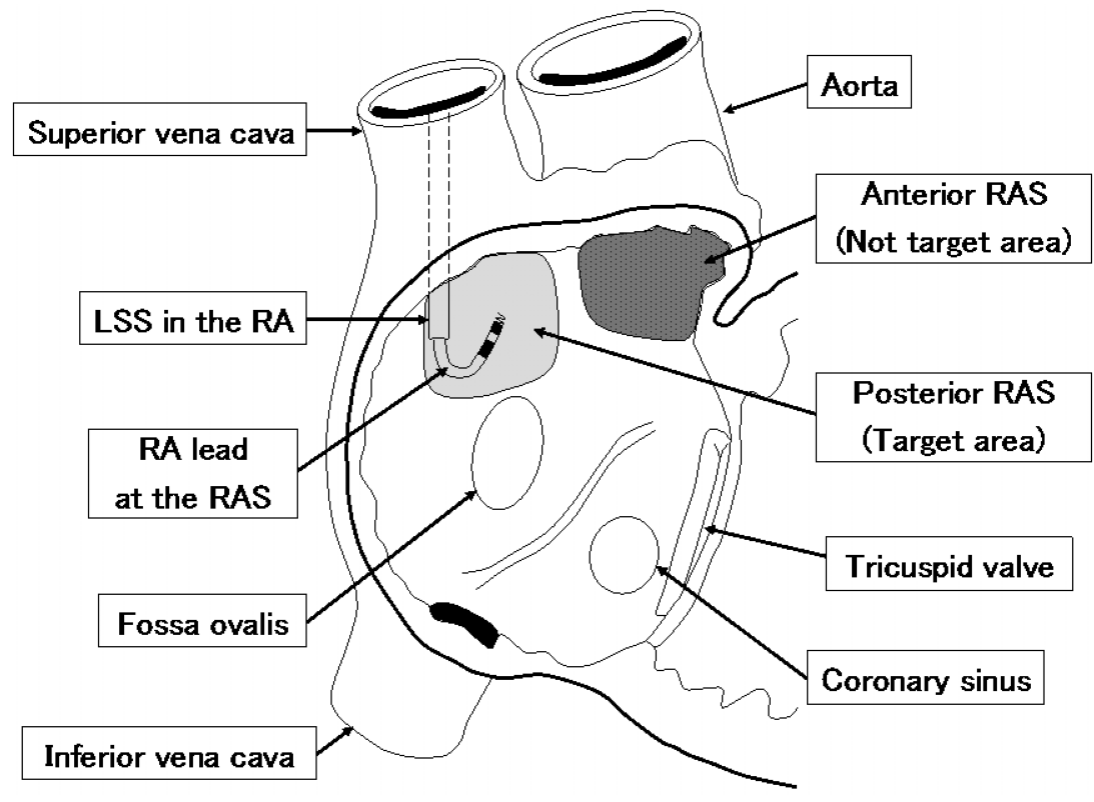

Fig 4. Target position is the posterior right atrial septum (RAS), which is near the superior vena cava and almost the same as that of posterior Backmann's bundle, but distant from the tricuspid valve. LSS, long-straight sheath; RA, right atrium.

\section{Statistical Analysis}

Data are presented as means \pm standard deviations. Differences in variables were assessed using Student's unpaired t-test or chi-square comparison. A p-value of less than 0.05 was considered significant.

\section{Results}

\section{Patient Characteristics}

The 76 patients comprised 39 patients with pacemakers, 30 patients with implantable cardioverter defibrillators (ICDs) and 7 patients undergoing cardiac resynchronization therapy. There were no significant differences between the patients in the non-LSS and LSS groups regarding their clinical backgrounds (Table 1).

\section{Success Rate}

The success rate of atrial lead insertion at the RAS was significantly higher in the LSS group than in the non-LSS group [50/52 (96.1\%) vs 13/24 (54.2\%); p<0.01].

Although the success rate for the last 5 patients and for the last 10 patients in the non-LSS group was $40 \%$, the success rate for the first 10 patients in the LSS group was $100 \%$.

In 2 patients in the LSS group, the atrial leads were dislodged at the time of peeling away the LSS, because of excessive pulling on the atrial lead. We repeated the process 
Table 1 Patient Characteristics

\begin{tabular}{lccc}
\hline \hline & Non-LSS group & LSS group & p value \\
\hline$n$ & 24 & 52 & \\
Age (years) & $60.4 \pm 11.5$ & $65.7 \pm 12.5$ & $N S$ \\
Men $(\%)$ & 70.8 & 75.8 & $N S$ \\
PM & 12 & 27 & $N S$ \\
SSS & 8 & 10 & $N S$ \\
AV block & 4 & 17 & $N S$ \\
ICD & 11 & 19 & $N S$ \\
IHD & 0 & 4 & $N S$ \\
DCM & 8 & 5 & $N S$ \\
HCM & 1 & 5 & $N S$ \\
Others & 2 & 5 & NS \\
\hline
\end{tabular}

LSS, long-straight sheath; PM, pacemaker; SSS, sick sinus syndrome; $A V$, atrioventricular; ICD, implantable cardioverter defibrillator; IHD, ischemic heart disease; DCM, dilated cardiomyopathy; HCM, hypertrophic cardiomyopathy; CRT, cardiac resynchronization therapy.

Table 2 Implantation Parameters in Patients With Successful Insertion at the RAS

\begin{tabular}{|c|c|c|c|}
\hline & Non-LSS group & LSS group & $p$ value \\
\hline$n$ & 13 & 50 & \\
\hline Implantation time (min) & $32.7 \pm 11.1$ & $13.6 \pm 9.1$ & $<0.01$ \\
\hline$P$ wave amplitude $(\mathrm{mV})$ & $2.9 \pm 1.4$ & $2.8 \pm 1.1$ & $N S$ \\
\hline Pacing threshold (V at $0.5 \mathrm{~ms}$ ) & $0.8 \pm 0.4$ & $0.8 \pm 0.4$ & $N S$ \\
\hline Lead impedance $(\Omega)$ & $608 \pm 183$ & $642 \pm 173$ & $N S$ \\
\hline$P$ wave duration during NSR $(\mathrm{ms})$ & $128 \pm 7$ & $125 \pm 11$ & $N S$ \\
\hline$P$ wave duration during RAS pacing ( $m s)$ & $116 \pm 14 *$ & $112 \pm 13 *$ & $N S$ \\
\hline
\end{tabular}

${ }^{*} p<0.05$ vs $P$-wave duration during NSR.

$R A S$, right atrial septum; NSR, normal sinus rhythm. Other abbreviation see in Table 1.

and were able to insert the leads at the RAS without a sheath, but the time required was longer than with the LSS.

The atrial lead was successfully inserted at the RAA in all of the 11 patients $(45.8 \%)$ in the non-LSS group in whom attempts at atrial lead insertion at the RAS failed.

\section{Operation Time}

Among the patients in whom atrial lead insertion at the RAS was successful (13 patients in the non-LSS group and 50 patients in the LSS group), implantation time was significantly shorter in patients in the LSS group than in patients in the non-LSS group $(13.6 \pm 9.1 \mathrm{~min}$ vs $32.7 \pm 11.1$ min, $\mathrm{p}<0.01)($ Table 2).

P-Wave Amplitude, Pacing Threshold and Lead Impedance Among the patients in whom atrial lead insertion at the RAS was successful (13 patients in the non-LSS group and 50 patients in the LSS group), there were no differences between the 2 groups in P-wave amplitude, pacing threshold or lead impedance (Table 2).

\section{P-Wave Duration on Surface ECG}

Among the patients in whom atrial lead insertion at the RAS was successful (13 patients in the non-LSS group and 50 patients in the LSS group), P-wave duration during NSR or RAS pacing on the 12-lead ECG did not differ between the 2 groups (Table 2). However, the P-wave duration was significantly shorter during RAS pacing than during NSR in both groups (non-LSS group: $116 \pm 14 \mathrm{~ms}$ vs $128 \pm 7 \mathrm{~ms}, \mathrm{p}<0.05$, LSS group: $112 \pm 13 \mathrm{~ms}$ vs $125 \pm 11 \mathrm{~ms}, \mathrm{p}<0.05$ ) (Table 2 ).

\section{Adverse Events}

No adverse cardiac events occurred during or after atrial lead implantation. Moreover, no FFRW sensing was observed with the atrial sensitivity threshold set to $0.3 \mathrm{mV}$ during either the operation or the follow-up period.

\section{Discussion}

We achieved a significantly higher success rate, a shorter implantation time of atrial lead insertion at the RAS, and shorter P-wave duration during RAS pacing, just by using the LSS.

\section{Development of the LSS Method}

Our initial technique of atrial lead insertion at the posterior RAS (posterior Bachmann's bundle) without using an LSS was very difficult to perform, and even when using a Locator $^{\mathrm{TM}}$ or a specially shaped stylet, it was still difficult to maintain the lead in a position perpendicular to the RAS, so the success rate was very low. Because the posterior RAS is very close to the superior vena cava, we could not obtain sufficient space for lead handling and the atrial leads could not be placed perpendicularly against the posterior RAS.

We then attempted lead insertion using a long tip-curved sheath to make direct contact with the RAS. Lead insertion using sheaths with various degrees of curvature was attempted, but it was still very difficult to keep the sheath and atrial lead perpendicular to the RAS, because the RAS has a sloped surface (Figs 3D-F). Consequently, the success rate of insertion using a long tip-curved sheath was less than $40 \%$. Therefore, we performed a simulation using a full-scale model of the heart (Path models, left to right heart in a travel case, Medtronic Inc) in order to determine which type of curved sheath was best for placing the atrial 
lead in a position perpendicular to the RAS. However, we gave up trying to use a tip-curved sheath in this position because of the difficulty in handling the sheath and atrial leads at the posterior RAS. However, during the simulation, we were able to obtain sufficient space for lead handling around the posterior RAS when using a LSS, with the tip located near the posterior free wall and distant from the RAS. Therefore, in our patients, we were able to obtain sufficient space for handling the atrial lead around the RAS, using this LSS method, and we achieved a higher success rate of implantation and shorter implantation time.

\section{Target Position of the Atrial Lead}

Various complications of atrial lead fixation at the RAA or free wall have been reported ${ }^{13-21}$ and in fact, considering the cardiac anatomy, it is surprising that perforation does not occur more frequently with atrial lead fixation at the RAA or free wall. Vlay et al reported that at the time of cardiac surgery, the tip of the helix of the right atrial electrode is occasionally visualized in patients with implanted pacemakers! ${ }^{17}, 18$ Oginosawa et al cited literature documenting $3(27 \%)$ of 11 autopsied pacemaker patients with penetration of the RA by the helix ${ }^{18}$ Greene et al reported that acute pericarditis occurred in $4.9 \%$ of patients with active fixation atrial leads, especially when the lead was placed at the lateral or anterolateral wall of the RA25 In addition, Weretka et al reported that FFRW sensing occurred in 10 $(21.3 \%)$ of 48 patients with a dual-chamber ICD26

We make an effort to avoid any free walls, such as the roof, posterior or RAA, for atrial lead fixation. We have attempted atrial lead insertion at the posterior RAS, the location of which is almost the same as that of Bachmann's bundle. Bailin et al reported a method of Bachmann's bundle pacing; ${ }^{23}$ however, this position is occasionally not the RAS but the anterior free wall and is near the aortic cusp, because it has been reported that atrial lead insertion at this position caused perforation or aortic injury? ${ }^{24}$ The low RAS should also be avoided because FFRW sensing frequently occurs? $22,27,28$ This "double counting" of intrinsic atrial deflection and FFRW sensing can not only interfere with the appropriate action of an ICD, ${ }^{27-29}$ but also result in an inappropriate mode switch in normal pacemaker patients 26,30-32 Therefore, these positions (ie, anterior free wall or low RAS) may cause operative complications.

Accordingly, the target position should be the posterior RAS (posterior Bachmann's bundle) and in the present no FFRW sensing occurred either during the operation or the follow-up period.

Unfortunately, because of insufficient data, we did not investigate whether posterior RAS pacing reduces the occurrence of PAF or not. However, the location of the posterior RAS is almost same as that of Bachmann's bundle and in fact, posterior RAS pacing significantly reduced the $\mathrm{P}$-wave duration compared with NSR, as has been the case with Bachmann's bundle pacing ${ }^{7}$ and low RAS pacing. ${ }^{27}$ This result can be attributed to preferential conduction from the RA to left atrium in the posterior RAS, ${ }^{33}$ and therefore posterior RAS pacing would potentially reduce the occurrence of PAF.

\section{Study Limitations}

First, this study was neither prospective nor randomized. We used the LSS in the second-term period, so there is a possibility that the higher success rate was related to the "learning curve". However, we experienced the following
2 cases in the LSS group in which we successfully inserted the atrial leads at the RAS in 5 and $7 \mathrm{~min}$, respectively. However, at the time of peeling away the LSS, the atrial leads were dislodged because of excessive pulling on the atrial leads. Therefore, we attempted to re-insert the leads at the RAS without the sheath, but it took approximately 30 and $35 \mathrm{~min}$, respectively, for fixation at the RAS. Thus, it took much longer to insert the atrial lead at the RAS without the LSS than with the LSS, even on the second attempt, which suggests the usefulness of the LSS with no relation to the "learning curve". In addition, although the success rate for the last 5 patients, and for the last 10 patients in the non-LSS group, was $40 \%$, the success rate for the first 10 patients in the LSS group was $100 \%$. The success rate increased dramatically after starting to use the LSS, which also suggests the usefulness of the LSS with no relation to the "learning curve".

Second, we used the LSS for atrial lead insertion at the RAS using a left-sided approach. However, in the rightsided approach, the tip of the LSS is near the septum and distant from the free wall, and sufficient space for handling of the lead near the RAS cannot be obtained. Therefore, the left-sided approach should be used for the LSS method.

\section{Conclusion}

We conclude that the LSS technique is very simple and useful for atrial lead insertion at the posterior RAS because space for lead handling can be secured and the tip of the sheath is near the free wall and distant from the RAS.

\section{Acknowledgments}

We are grateful to Miyuki Fujiwara, Mami Ohgiri, Masaharu Fujita, Hidehiro Hachiya, Motohiko Imura and Kiyoshi Okano for their excellent technical assistance.

\section{References}

1. Rosenqvist M, Brandt J, Schuller H. Long-term pacing in sinus node disease: Effects of stimulation mode on cardiovascular morbidity and mortality. Am Heart J 1988; 116: 16-22.

2. Stangl K, Seitz K, Wirtzfeld A, Alt E, Blomer H. Differences between atrial single chamber pacing (AAI) and ventricular single chamber pacing (VVI) with respect to prognosis and antiarrhythmic effect in patients with sick sinus syndrome. Pacing Clin Electrophysiol 1990; 13: 2080-2085.

3. Brandt J, Anderson H, Fahraeus T, Schuller H. Natural history of sinus node disease treated with atrial pacing in 213 patients: Implications for selection of stimulation mode. J Am Coll Cardiol 1992; 20: 633 639.

4. Andersen HR, Thuesen L, Bagger JP, Vesterlund T, Thomsen PE. Prospective randomised trial of atrial versus ventricular pacing in sicksinus syndrome. Lancet 1994; 344: 1523-1528.

5. Ricci R, Pignalberi C, Santini L, Magris B, Russo M, Grovale N, et al. Physiologic pacing for atrial fibrillation prevention in sinus node disease: Long-term results. Pacing Clin Electrophysiol 2006; 29 (Suppl): S54-S60.

6. Sgarbossa EB, Pinski SL, Maloney JD, Simmons TW, Wilkoff BL, Castle LW, et al. Chronic atrial fibrillation and stroke in paced patients with sick sinus syndrome: Relevance of clinical characteristics and pacing modalities. Circulation 1993; 88: 1045-1053.

7. Bailin SJ, Adler S, Giudici M. Prevention of chronic atrial fibrillation by pacing in the region of Bachmann's bundle: Results of a multicenter randomized trial. J Cardiovasc Electrophysiol 2001; 12: 912-917.

8. Padeletti L, Michelucci A, Pieragnoli P, Colella A, Musilli N. Atrial septal pacing: A new approach to prevent atrial fibrillation. Pacing Clin Electrophysiol 2004; 27: 850-854.

9. Padeletti L, Purerfellner H, Adler SW, Waller TJ, Harvey M, Horvitz L, et al. Combined efficacy of atrial septal lead placement and atrial pacing algorithms for prevention of paroxysmal atrial tachyarrhythmia. J Cardiovasc Electrophysiol 2003; 14: 1189-1195.

10. Kale M, Bennett DH. Pacemaker prevention therapies for the control 
of drug-refractory paroxysmal atrial fibrillation. Europace 2003; 5: $123-131$.

11. Padeletti L, Pieragnoli P, Ciapetti C, Colella A, Musilli N, Porciani $\mathrm{MC}$, et al. Randomized crossover comparison of right atrial appendage pacing versus interatrial septum pacing for prevention of paroxysmal atrial fibrillation in patients with sinus bradycardia. Am Heart J 2001; 142: $1047-1055$.

12. Manolis AG, Katsivas AG, Vassilopoulos C, Koutsogeorgis D, Louvros NE. Prevention of atrial fibrillation by inter-atrial septum pacing guided by electrophysiological testing, in patients with delayed interatrial conduction. Europace 2002; 4: 165-174.

13. Dilling-Boer D, Ector H, Willems R, Heidbuchel H. Pericardial effusion and right-sided pneumothorax resulting from an atrial active-fixation lead. Europace 2003; 5: 419-423.

14. Khan MN, Joseph G, Khaykin Y, Ziada KM, Wilkoff BL. Delayed lead perforation: A disturbing trend. Pacing Clin Electrophysiol 2005; 28: 251-253.

15. Velavan P, Chauhan A. An unusual presentation of delayed cardiac perforation caused by atrial screw-in lead. Heart 2003; 89: 364 .

16. Ellenbogen KA, Wood MA, Shepard RK. Delayed complications following pacemaker implantation. Pacing Clin Electrophysiol 2002; 25: $1155-1158$.

17. Vlay SC. Complications of active-fixation electrodes. Pacing Clin Electrophysiol 2002; 25: 1153-1154.

18. Oginosawa $\mathrm{Y}, \mathrm{Abe} \mathrm{H}$, Nakashima Y. Right pneumothorax resulting from an endocardial screw-in atrial lead in an implantable cardioverter defibrillator system. Pacing Clin Electrophysiol 2002; 25: 1278-1279.

19. Ho WJ, Kuo CT, Lin KH. Right pneumothorax resulting from an endocardial screw-in atrial lead. Chest 1999; 116: 1133-1134.

20. Aizawa K, Kaneko Y, Yamagishi T, Utsugi T, Suzuki T, Ishikawa S, et al. Oozing from the pericardium as an etiology of cardiac tamponade associated with screw-in atrial leads. Pacing Clin Electrophysiol 2001; 24: 381-383.

21. Sivakumaran S, Irwin ME, Gulamhusein SS, Senaratne MP. Postpacemaker implant pericarditis: Incidence and outcomes with active-fixation leads. Pacing Clin Electrophysiol 2002; 25: 833-837.

22. de Voogt WG, van Mechelen R, van den Bos AA, Scheffer M, van Hemel NM, Levine PA. Electrical characteristics of low atrial septum pacing compared with right atrial appendage pacing. Europace 2005;
7: 60-66.

23. Bailin SJ. Atrial lead implantation in the Bachmann bundle. Heart Rhythm 2005; 2: 784-786.

24. Kashani A, Mehdirad A, Fredman C, Barold SS. Aortic perforation by active-fixation atrial pacing lead. Pacing Clin Electrophysiol 2004; 27: 417-418.

25. Greene TO, Portnow AS, Huang SK. Acute pericarditis resulting from an endocardial active fixation screw-in atrial lead. Pacing Clin Electrophysiol 1994; 17: 21-25.

26. Weretka S, Becker R, Hilbel T, Karle C, Osswald BR, Kuebler W, et al. Far-field $\mathrm{R}$ wave oversensing in a dual chamber arrhythmia management device: Predisposing factors and practical implications. Pacing Clin Electrophysiol 2001; 24: 1240-1246.

27. De Voogt WG, Van Mechelen R, Van Den Bos A, Scheffer M, Van Hemel NM, Koistinen J. A technique of lead insertion for low atrial septal pacing. Pacing Clin Electrophysiol 2005; 28: 639-646.

28. Inama G, Santini M, Padeletti L, Boriani G, Botto G, Capucci A, et al. Far-field $\mathrm{R}$ wave oversensing in dual chamber pacemakers designed for atrial arrhythmia management: Effect of pacing site and lead tip to ring distance. Pacing Clin Electrophysiol 2004; 27: 1221-1230.

29. Acosta H, Pothula VR, Rodriguez M, Ramadas S, Castellanos A. Placement of a pacing lead at the inferior portion of the interatrial septum without special tools. Pacing Clin Electrophysiol 2007; 30 (Suppl 1): S84-S87.

30. Brandt J, Fahraeus T, Schuller H. Far-field QRS complex sensing via the atrial pacemaker lead. II: Prevalence, clinical significance and possibility of intraoperative prediction in DDD pacing. Pacing Clin Electrophysiol 1988; 11: 1540-1544.

31. Bordacher P, Cazeau S, Graindorg L, Ritter P. Impact and prevention of far-field sensing in fallback mode switches. Pacing Clin Electrophysiol 2003; 26: 206-209.

32. de Voogt WG, van Hemel NM, van de Bos AA, Koistinen J, Fast JH. Verification of pacemaker automatic mode switching for the detection of atrial fibrillation and atrial tachycardia with Holter recording. Europace 2006; 8: 950-961.

33. Roithinger FX, Cheng J, SippensGroenewegen A, Lee RJ, Saxon LA, Scheinman MM, et al. Use of electroanatomic mapping to delineate transseptal atrial conduction in humans. Circulation 1999; 100: $1791-1797$. 